\title{
Generalized analysis of the direct weakly interacting massive particle searches
}

\author{
Andriy Kurylov and Marc Kamionkowski \\ California Institute of Technology, Pasadena, California 91125, USA
}

(Received 23 July 2003; published 10 March 2004)

\begin{abstract}
We perform a generalized analysis of data from WIMP search experiments for pointlike WIMPs of arbitrary spin and general Lorenz-invariant WIMP-nucleus interaction. We explicitly show that in the nonrelativistic limit only spin-independent (SI) and spin-dependent (SD) WIMP-nucleon interactions survive, which can be parametrized by only five independent parameters. We explore this five-dimensional parameter space to determine whether the annual modulation observed in the DAMA experiment can be consistent with all other experiments which reported null results. The pure SI interaction is ruled out except for a very small region of parameter space with the WIMP mass close to $50 \mathrm{GeV}$ and the ratio of the WIMP-neutron to WIMP-proton SI couplings $-0.77 \leqslant f_{n} / f_{p} \leqslant-0.75$. For the predominantly SD interaction, we find an upper limit on the WIMP mass of about $18 \mathrm{GeV}$, which can only be weakened if the constraint stemming from null searches for energetic neutrinos from WIMP annihilation the Sun is evaded. None of the regions of the parameter space that can reconcile all WIMP search results can be easily accommodated in the minimal supersymmetric standard model.
\end{abstract}

DOI: 10.1103/PhysRevD.69.063503

PACS number(s): 95.35.+d

\section{INTRODUCTION}

The existence of dark matter that accounts for roughly a third of the energy density of the Universe is strongly supported by existing astrophysical observations. The origin of dark matter is as of yet unknown, and suggested candidates include massive compact halo objects (MACHOs), massive neutrinos, axions, weakly interacting massive particles (WIMPs), Kaluza-Klein excitations of standard model particles, etc. (see Ref. [1] for a brief review). Out of the above, the WIMP is one of the most natural candidates since for such a particle with a mass of about $100 \mathrm{GeV}$ one easily obtains the required present energy density without much fine-tuning.

WIMPs can be detected directly via observation of nuclear recoils in low background detectors and indirectly via observation of their annihilation products, such as high energy neutrinos or charged leptons, coming from the Sun or Earth. The latter method is much more model dependent and requires either that the WIMP be a self-charged-conjugate particle or that there be both WIMPs and anti-WIMPs available at present with sufficient abundances.

In direct searches a WIMP is assumed to scatter from a nucleus via some combination of spin-independent (SI) and spin-dependent (SD) interactions. In the former case, the scattering amplitude scales with the mass of the nucleus, whereas in the latter it exists only for nuclei with spin and is often dominated by the WIMP SD interaction with the unpaired nucleon. Part of the nuclear recoil energy is spent on ionizing the detector medium and light emitted during recombination is used as a signal. Using such a technique, the DAMA Collaboration has reported an annual modulation signal in their $\mathrm{NaI}$ detector [2] compatible with the variation in the flux of WIMPs incident on the detector due to the motion of Earth through the galactic halo. When interpreted in terms of the SI WIMP-nucleon interaction, the DAMA measurements resulted in the preferred region in the WIMP cross section versus WIMP mass plane. This region was, however, almost entirely excluded by the results reported by the
CDMS [3] and subsequently EDELWEISS [4] and ZEPLIN-I [5] Collaborations. Interpretation of the DAMA result in terms of a purely SD WIMP-nucleon interaction has been studied in Ref. [6]. It has been shown there that such an interpretation is inconsistent with the constraints set by the experiment with an enriched liquid Xe detector [7] and with indirect constraints coming from nonobservation of high energy upward muons reported by the Super-Kamiokande (SK) Collaboration [8]. In the latter case, the muons are created in the Earth by the neutrinos produced in WIMP annihilation in the Sun.

In this work we perform a model-independent analysis of data from the above direct search experiments. We show that the interaction of a heavy particle ${ }^{1}$ with a nucleon can in general be described by only five parameters: its mass $M_{\chi}$, its SI and SD cross sections on the proton $\sigma_{\chi p}^{S I}$ and $\sigma_{\chi p}^{S D}$, and the ratios of the SI and SD couplings to the proton and neutron, $f_{n} / f_{p}$ and $a_{n} / a_{p}$. We vary all five parameters without any constraints that may appear in specific models [such as $f_{n} \approx f_{p}$ in the minimal supersymmetric standard model (MSSM) [9]]. We also allow for the possibility that the WIMP is not self-conjugate, in which case the SK constraint might be evaded.

We find several regions in the five-parameter space where the results in Refs. [2-4,7] can be reconciled. ${ }^{2}$ If the SK constraint does not apply, it is easy to bring all data in agreement with the predominantly SD WIMP-proton interaction. Here, the SD WIMP-neutron interaction and SI WIMPnucleon interactions are also allowed. If the SK constraint does apply, then a substantial SD WIMP-neutron coupling is required, with an upper limit on the WIMP mass of about 18

\footnotetext{
${ }^{1}$ Here, we for simplicity always refer to such a particle as a WIMP.

${ }^{2}$ Uncertainties in astrophysical inputs should in principle be considered in determining precise shapes of the regions (see, e.g., Ref. [10] and references therein for discussion). Such a detailed study, which would require access to DAMA data, is beyond the scope of this work.
} 
$\mathrm{GeV}$. These results are similar to the findings reported in Ref. [6]. The upper limit on the WIMP mass is slightly improved, however, with the inclusion of ZEPLIN-I results. We also find another solution for the predominantly SI interaction with $M \chi \sim 50 \mathrm{GeV}$ and $-0.76<f_{n} / f_{p}<-0.74$. However, in this case the agreement is marginal and requires finetuning.

Although the above solutions are allowed from a purely phenomenological point of view, none of them is natural in the MSSM. For instance, the existing lower limit on the WIMP mass within the MSSM is $37 \mathrm{GeV}$ [1]. This limit is not completely general but evading it, if at all possible, will require substantial fine-tuning of the MSSM parameters. The difficulties in obtaining the required parameters for each solution within the MSSM are discussed in detail in the text. Note, however, that if the DAMA result is removed from the analysis, the MSSM can account for all other experiments and still produce a sufficient abundance of WIMPs to account for all dark matter (see Ref. [1]).

This paper is organized as follows. After briefly reviewing the theoretical aspects of WIMP-nucleus elastic scattering in Sec. II we describe the procedures we used to analyze various data sets in Sec. IV and present our analysis in Sec. V. We conclude in Sec. VI.

\section{GENERALIZED WIMP-NUCLEON INTERACTION}

WIMP searches have usually been interpreted within the framework of the MSSM because it contains an excellent WIMP candidate, the lightest neutralino. Interactions of the neutralino with quarks (and ultimately with nucleons and nuclei) have been extensively studied in the literature (see, e.g., $[9,11])$. In the nonrelativistic limit and as a result of the Majorana nature of the neutralino, the neutralino-nucleon interaction has only two terms

$$
\begin{aligned}
\mathcal{L}_{\chi} & =\bar{\chi} \gamma^{\mu} \gamma_{5} \chi \mathcal{J}_{\mu}^{5}(x)+\bar{\chi} \chi \mathcal{S}(x), \\
\mathcal{J}_{\mu}^{5}(x) & =\sqrt{2} G_{F}\left(a_{p} \bar{p} \gamma_{\mu} \gamma_{5} p+a_{n} \bar{n} \gamma_{\mu} \gamma_{5} n\right), \\
\mathcal{S}(x) & =f_{p} \bar{p} p+f_{n} \bar{n} n,
\end{aligned}
$$

where $G_{F}$ is the Fermi constant, and $a_{p, n}$ and $f_{p, n}$ are, respectively, the proton and neutron coupling constants given in Ref. [9]. ${ }^{3}$ The term containing $\mathcal{S}(x)$ gives rise to the SI interaction, whereas the $\mathcal{J}_{\mu}^{5}(x)$ term is responsible for the SD interaction.

Although Eq. (1) was derived assuming that WIMPs are heavy Majorana spin-1/2 fermions within the MSSM, it can be generalized to particles of arbitrary spin, independent of whether they are self-conjugate. Consider a general Lorentzinvariant interaction for the WIMP-nucleon coupling:

\footnotetext{
${ }^{3}$ We absorbed the coupling constants into the definitions of $\mathcal{J}_{\mu}^{5}$ and $\mathcal{S}$ to make this simple notation consistent with the convention adopted in Ref. [9].
}

$$
\begin{aligned}
\mathcal{L}_{\chi}= & \left(\mathcal{S}_{\chi}+\mathcal{P}_{\chi}\right)\left(G_{s} \bar{N} N+G_{p} \bar{N} \gamma_{5} N\right)+\left(\mathcal{V}_{\chi}^{\mu}+\mathcal{A}_{\chi}^{\mu}\right) \\
& \times\left(G_{v} \bar{N} \gamma_{\mu} N+G_{a}\left\{\bar{N} \gamma_{\mu} \gamma_{5} N+2 M_{N} \frac{q_{\mu}}{m_{\pi}^{2}-q^{2}} \bar{N} \gamma_{5} N\right\}\right) \\
& +\left(\mathcal{T}_{\chi}^{\mu \nu}+\mathcal{D}_{\chi}^{\mu \nu}\right)\left(G_{t} \bar{N} \sigma_{\mu \nu} N+G_{d} \bar{N} \sigma_{\mu \nu} \gamma_{5} N\right)
\end{aligned}
$$

Here $\mathcal{S}_{\chi}$ and $\mathcal{P}_{\chi}$ are linear combinations of scalar and pseudoscalar operators built from the $\chi$ field, $\mathcal{V}_{\chi}^{\mu}$ and $\mathcal{A}_{\chi}^{\mu}$ are the corresponding vector and axial-vector operators, and $\mathcal{T}_{\chi}^{\mu \nu}$ and $\mathcal{D}_{\chi}^{\mu \nu}$ are the tensor and pseudotensor operators. In Weyl representation, the Dirac structures appearing in Eq. (2) are (Latin indices indicate spatial components)

$$
\begin{aligned}
\gamma^{0} & =\left(\begin{array}{ll}
0 & 1 \\
1 & 0
\end{array}\right), \quad \gamma^{i}=\left(\begin{array}{cc}
0 & \sigma^{i} \\
-\sigma^{i} & 0
\end{array}\right), \quad \gamma_{5}=\left(\begin{array}{cc}
-1 & 0 \\
0 & 1
\end{array}\right), \\
\sigma^{\mu \nu} & =\frac{i}{2}\left\{\gamma^{\mu} \gamma^{\nu}-\gamma^{\nu} \gamma^{\mu}\right\},
\end{aligned}
$$

where $\sigma^{i}$ are Pauli matrices. In order to use the above interaction to calculate matrix elements for WIMP-nucleus scattering one must sum over all nucleons in the target nucleus.

The WIMP-nucleon interaction is determined by the underlying WIMP-quark interaction, and to obtain Eq. (2) from the underlying interaction one must take its matrix elements between one-nucleon states. In the limit $|\vec{q}| \ll M_{N}$ one can do this by simply replacing the quark fields with the nucleon fields and rescaling the corresponding coupling constants. For example, $g_{V}^{q}\left\langle N\left|\bar{q} \gamma_{\mu} q\right| N\right\rangle=G_{V}^{q} \bar{u}_{N} \gamma_{\mu} u_{N}$, where $u_{N}$ is the nucleon spinor. This procedure holds for all operators except for quark axial-vector current whose matrix element has a pole:

$$
\begin{aligned}
\left\langle N\left|J_{q 5}^{\mu}\right| N\right\rangle & =\bar{N}\left(g_{A} \gamma^{\mu} \gamma^{5}+g_{P}\left(q^{2}\right) q^{\mu} \gamma_{5}\right) N, \\
g_{P}\left(q^{2}\right) & =g_{A} \frac{2 M_{N}}{m_{\pi}^{2}-q^{2}}
\end{aligned}
$$

where the second line implements the partial conservation of axial-vector current (PCAC) hypothesis and $m_{\pi}$ is the pion mass. This argument justifies the appearance of the particular structure multiplying $G_{a}$ in Eq. (2). The second term in the first line of Eq. (4) becomes important if $|\vec{q}| \geqslant m_{\pi}$ [11]. For simplicity, we do not explicitly keep the pion pole term in the following. However, it should be understood that this term always appears in the form given by the last term in the second line of Eq. (2).

Except for the pion pole term, Eq. (2) does not contain operators with explicit factors of momentum transfer $q$. Such terms are considered subleading. As will be shown below, such operators are suppressed by powers of $q / M_{p}$ or $q / M_{\chi}$. It is easy to show that in the frame where the nucleus is initially at rest the components of $q$ are related by $2 q_{0} M_{n u c}+q^{2}=0$, which also shows that $q_{0} \ll|\vec{q}|$. Therefore, we find 


$$
\frac{q}{M_{p}} \sim \sqrt{\frac{q_{0} M_{n u c}}{M_{p}^{2}}}=\sqrt{\frac{q_{0} A}{M_{p}}} \lesssim 0.01 \sqrt{A} \lesssim 0.1,
$$

where $A \lesssim 100$ is the atomic number of the nucleus and $q_{0}$ is the recoil energy, which we assume not to significantly exceed $100 \mathrm{keV}$.

Let us look at each of the one-nucleon operators appearing in Eq. (2) and determine which of them have nonvanishing nuclear matrix elements in the nonrelativistic limit.

Scalar operators. The scalar operator $\bar{N} N$ simply counts nucleons; it obviously survives in the nonrelativistic limit. On the other hand, nuclear matrix elements of the pseudoscalar operator $\mathcal{P}_{N}=\Sigma_{k} \bar{N}_{k}\left(x+r_{k}\right) \gamma_{5} N_{k}\left(x+r_{k}\right)$ are suppressed: $\left\langle f\left|\mathcal{P}_{N}\right| i\right\rangle \sim|\vec{q}| / M_{p}$. Here, $x$ and $r_{k}$ correspond, respectively, to the center of mass of the nucleus and to the position of the $k$ th nucleon relative to the center of mass. Since our discussion is not affected by the fact that the nucleus has a finite size, we can consider it a point particle and set all $r_{k}$ to zero. In a more accurate treatment one can account for finite size $R$ by introducing a form factor $F(|\vec{q}| R)$. Defining an "axial-vector current" operator $\hat{A}_{5}^{\mu}(x)=\int{ }^{x} \mathcal{P}_{N}(x) d x^{\mu}$ as an intermediate step we obtain

$$
\begin{aligned}
\left\langle f\left|\mathcal{P}_{N}(x)\right| i\right\rangle & =\left\langle f\left|\partial_{\mu} \hat{A}_{5}^{\mu}(x)\right| i\right\rangle=i q_{\mu} e^{i q \cdot x}\left\langle f\left|\hat{A}_{5}^{\mu}(0)\right| i\right\rangle \\
& \approx i q_{i} e^{i q \cdot x} P\left\langle f\left|\hat{J}_{N}^{i}\right| i\right\rangle+\mathcal{O}\left(q^{2}\right)
\end{aligned}
$$

where $\hat{J}_{N}$ is the nuclear spin operator. In the last step we used the fact that spin is the only axial vector describing a state of the nucleus that does not vanish as $q \rightarrow 0$. The scalar constant $P$ has the dimension of inverse mass. The largest quantity that has this property and is not singular in the limit $q \rightarrow 0$ is the inverse mass of the nucleon. Therefore, $P \sim 1 / M_{p}$, and matrix elements of $\mathcal{P}_{N}$ are suppressed according to Eq. (5). Another way to see that $P \sim 1 / M_{p}$ (and, e.g., not $1 / M_{n u c}$ ) is to first consider the one-nucleon operator $\bar{N} \gamma_{5} N$ using free Dirac spinors for the nucleon fields. Using free spinors is a good approximation because the binding energy of a nucleon in a nucleus is much smaller than the nucleon mass, which means that nucleons are only slightly off shell. A direct calculation yields

$$
\bar{N}(p+q) \gamma_{5} N(p)=\frac{q_{i}}{M_{p}} \eta^{\dagger^{\prime}} \sigma_{i} \eta
$$

where $\eta, \eta^{\prime}$ are Weyl spinors characterizing the states of the nucleon with the initial momentum $p$ and $\sigma_{i}$ is the vector of Pauli matrices. In a nucleus one obtains

$$
\frac{q_{i}}{M_{p}}\left\langle f\left|\sum_{k} \eta_{k}^{\dagger^{\prime}} \sigma_{i} \eta_{k}\right| i\right\rangle \sim \frac{q_{i}}{M_{p}}\left\langle f\left|\hat{J}_{N}^{i}\right| i\right\rangle,
$$

in agreement with Eq. (6).

Vector operators. For $\mathcal{V}_{N}^{\mu}=\Sigma_{k} \bar{N}_{k} \gamma^{\mu} N_{k}$ we must have

$$
\left\langle f\left|\mathcal{V}_{N}^{\mu}\right| i\right\rangle=V_{N}^{+}\left(p_{f}+p_{i}\right)^{\mu}+V_{N}^{-}\left(p_{f}-p_{i}\right)^{\mu}
$$

where $V_{N}^{+}, V_{N}^{-}$are scalar constants. Here, $p_{i}=\left(M_{n u c}, \overrightarrow{0}\right)$ and $p_{f}=q+p_{i}$. Just like for $\mathcal{P}_{N}$, we may conclude on dimensional grounds that $V_{N}^{+}, V_{N}^{-} \sim 1 / M_{p}$. Then the time component of $\mathcal{V}_{N}$ is of order unity, whereas the spatial components are suppressed: $\mathcal{V}_{N}^{i} \sim q^{i} / M_{p}$. The time component $\mathcal{V}_{N}^{0}$ multiplies $\mathcal{V}_{\chi, 0}$ and $\mathcal{A}_{\chi, 0}$ in Eq. (2), which transform, respectively, as a scalar and a pseudoscalar under the extended rotational group. Therefore, they can be effectively absorbed into $\mathcal{S}_{\chi}$ and $\mathcal{P}_{\chi}$ in the nonrelativistic case.

For the axial-vector operator $\mathcal{A}_{N 5}^{\mu}=\Sigma_{k} \bar{N}_{k} \gamma^{\mu} \gamma_{5} N_{k}$ the situation is reversed. The time component transforms as a pseudoscalar under the extended rotational group and its matrix elements are suppressed for the same reason as those of $\mathcal{P}_{N}$. The spatial components transform like a pseudovector leading to

$$
\left\langle f\left|\mathcal{A}_{N 5}^{i}\right| i\right\rangle=A_{N}\left\langle f\left|\hat{J}_{N}^{i}\right| i\right\rangle,
$$

where $A_{N}$ is a dimensionless constant, which can in general be of order unity.

Tensor operators. Let us first consider the operator $\mathcal{T}_{N}^{\mu \nu}$ $=\Sigma_{k} \bar{N}_{k} \sigma^{\mu \nu} N_{k}$. Nonzero components of $\sigma^{\mu \nu}$ are $\sigma^{0 i}$ and $\sigma^{i j}, i, j=1,2,3$. Under the extended rotational group, $\sigma^{0 i}$ transforms as a polar vector. Therefore, in analogy to Eq. (9),

$$
\mathcal{T}_{N}^{0 i}=T_{N}^{+}\left(p_{f}+p_{i}\right)^{i}+T_{N}^{-}\left(p_{f}-p_{i}\right)^{i},
$$

which is suppressed similarly to $\mathcal{V}_{N}^{i}$ in Eq. (9). To analyze $\mathcal{T}_{N}^{i j}$ consider its dual $\mathcal{T}_{N}^{i}=\epsilon^{i j k} \mathcal{T}_{N, j k}=\Sigma_{k} \bar{N}_{k} \epsilon^{i j k} \sigma_{j k} N_{k}$ $\equiv 2 \Sigma_{k} \bar{N}_{k} \sigma^{i} N_{k}$, where $\epsilon^{i j k}$ is the three-dimensional LeviCività tensor. In the last step, we used the property $\sigma_{j k}$ $=\epsilon^{j k l} \sigma_{l}$, which can be derived from Eq. (3). We obtain

$$
\begin{aligned}
\left\langle f\left|\mathcal{T}_{N}^{i j}\right| i\right\rangle & =\frac{1}{2} \epsilon^{i j k} \mathcal{T}_{N, j}=\epsilon^{i j k}\left\langle f\left|\sum_{k} \bar{N}_{k} \sigma^{i} N_{k}\right| i\right\rangle \\
& =T_{N} \epsilon^{i j k}\left\langle f\left|\hat{J}_{N, k}\right| i\right\rangle,
\end{aligned}
$$

where $T_{N}$ is a dimensionless constant. Therefore, $\mathcal{T}_{N}^{i j}$ survives in the nonrelativistic limit.

The operator $\mathcal{D}_{N}^{\mu \nu}=\sum_{k} \bar{N}_{k} \sigma^{\mu \nu} \gamma_{5} N_{k}$ can be expressed as $(i / 2) \epsilon^{\mu \nu \rho \lambda} \mathcal{T}_{N, \rho \lambda}$, which follows from the identity $\sigma^{\mu \nu} \gamma_{5}$ $=(i / 2) \epsilon^{\mu \nu \rho \lambda} \sigma_{\rho \lambda}$. Since only $\mathcal{T}_{N}^{i j}$ survives in the nonrelativistic limit, we conclude that $\mathcal{D}_{N}^{0 i}$ are the only nonvanishing components of $\mathcal{D}_{N}^{\mu \nu}$ in this limit:

$$
\mathcal{D}_{N}^{0 i}=\frac{i}{2} \epsilon^{0 i j k} \mathcal{T}_{N, j k}=\frac{i}{4} \epsilon^{0 i j k} \epsilon_{j k l} \mathcal{T}_{N}^{l}=\frac{i T_{N}}{2}\left\langle f\left|\hat{J}_{N}^{i}\right| i\right\rangle
$$

The antisymmetric tensor $\epsilon_{i j k}$ in Eq. (12) is contracted with spatial components of $\mathcal{T}_{\chi}^{\mu \nu}$ and $\mathcal{D}_{X}^{\mu \nu}$ in Eq. (2). The corresponding quantities $\mathcal{T}_{\chi}^{i j} \epsilon_{i j k}$ and $\mathcal{D}_{\chi}^{i j} \epsilon_{i j k}$ transform, respectively, as an axial and a polar vector under the extended rotational group. Therefore, in the nonrelativistic limit $\mathcal{T}_{\chi}^{i j} \epsilon_{i j k}$ can be absorbed into $\mathcal{A}_{\chi, k}$ and $\mathcal{D}_{\chi}^{i j} \epsilon_{i j k}$ into $\mathcal{V}_{\chi, k}$. Similarly, taking Levi-Cività structure $\epsilon^{0 i j k} \epsilon_{j k l}$ from Eq. (13) we conclude that $\mathcal{T}_{\chi, 0 i} \epsilon^{0 i j k} \epsilon_{j k l} \equiv-2 \mathcal{T}_{\chi, 0 l}$ transforms as a po- 
lar vector and $\mathcal{D}_{\chi, 0 i} \epsilon^{0 i j k} \epsilon_{j k l} \equiv-2 \mathcal{D}_{\chi, 0 l}$ as an axial vector. They can be absorbed into, respectively, $\mathcal{V}_{\chi, l}$ and $\mathcal{A}_{\chi, l}$. With the above considerations in mind Eq. (2) can be effectively rewritten as follows in the nonrelativistic limit:

$$
\mathcal{L}_{\chi}=\left(\mathcal{S}_{\chi}+\mathcal{P}_{\chi}\right) G_{s} \bar{N} N+\left(\mathcal{V}_{\chi}^{i}+\mathcal{A}_{\chi}^{i}\right) G_{a} \bar{N} \gamma_{i} \gamma_{5} N
$$

where $\mathcal{S}_{\chi}, \mathcal{P}_{\chi}, \mathcal{V}_{\chi}^{i}$, and $\mathcal{A}_{\chi}^{i}$ have been redefined to absorb the contributions from the vector and tensor interactions. Moreover, since the general arguments used for $\mathcal{P}_{N}$ and $\mathcal{V}_{N}^{i}$ also apply to $\mathcal{P}_{\chi}$ and $\mathcal{V}_{\chi}^{i}$ (with the suppression $|\vec{q}| / M_{\chi}$ $\sim 10^{-3}$ in this case), we can safely neglect the latter.

The scalar density $\mathcal{S}_{\chi}(x)$ in Eq. (14) can be written as $\chi^{\dagger} \chi$. For a WIMP of spin $J_{\chi}, \chi$ is a $\left(2 J_{\chi}+1\right)$-component nonrelativistic spinor (e.g., in the MSSM, $\chi$ is a twocomponent Weyl spinor). The spatial components of the axial-vector density $\mathcal{A}_{\chi}^{l}(x)$ in the nonrelativistic limit are proportional to the spin density $\chi^{\dagger} \hat{S}_{\chi}^{l} \chi$. Finally, we arrive at the following form of the WIMP-nucleon interaction Lagrangian in the nonrelativistic limit:

$$
\begin{aligned}
\mathcal{L}_{\chi}= & 4 f_{N} \chi_{s}^{\dagger} \chi_{s} \eta_{N}^{\dagger} \eta_{N}+16 \sqrt{2} G_{F} a_{N} \chi^{\dagger} \vec{S}_{\chi} \chi \eta_{N}^{\dagger} \vec{S}_{N} \eta_{N} \\
& +\mathcal{O}\left(\frac{q}{M_{p, \chi}}\right)
\end{aligned}
$$

where $\eta_{N}$ is the two-component Weyl spinor for the nucleon (initial and final state spinors may be different), $N=n, p$, $\vec{S}_{N}=\vec{\sigma} / 2$, and the notation for the couplings has been adjusted to match that of Eq. (1). In this form, Eq. (15) is valid for nonrelativistic pointlike WIMPs of arbitrary spin.

The operators neglected in Eq. (15) are suppressed by a factor of $0.01 \sqrt{A}$ relative to the leading order terms [see Eq. (5)]. Strictly speaking, in neglecting such terms we made an implicit assumption that the couplings $A \cdot f_{N}$ and $G_{F} a_{N}$ in Eq. (15) are not suppressed relative to the other couplings in Eq. (2), such as $G p$. If it turned out that $G p \gtrsim 100 \sqrt{A} f_{N}$, then one would have to consider the $\bar{N} \gamma_{5} N$ operator even though it is formally suppressed in the nonrelativistic limit. In this paper we ignore this possibility, and modulo the aforementioned assumption our analysis is model-independent.

\section{Some examples}

Below we consider some examples theories that have WIMPS with spin 0, spin 1/2 (Dirac particle), and spin 1 and explicitly show that in each case the WIMP-nucleon interaction reduces to Eq. (15).

\section{Spin 0}

It is possible that in a supersymmetric theory the lightest supersymmetric (SUSY) particle is the scalar partner of the neutrino, $\widetilde{\nu}$. In the nonrelativistic limit, such a particle interacts with a nucleon as follows [12]:

$$
\mathcal{L}_{\tilde{\nu}}=-\sqrt{2} G_{F} J_{\tilde{\nu}}^{\mu} J_{N, \mu}
$$

$$
\begin{aligned}
& J_{\tilde{\nu}}^{\mu}=\left(p_{\nu}^{\prime \mu}+p_{\nu}^{\mu}\right) \tilde{\nu}_{\nu}^{\dagger} \tilde{\nu}, \\
& J_{N}^{\mu}=\left(T_{N}^{3}-2 Q_{N} \sin ^{2} \theta_{W}\right) \bar{N} \gamma^{\mu} N,
\end{aligned}
$$

where $p_{\tilde{\nu}}^{\mu}, p_{\tilde{\nu}}^{\prime \mu}$ are the initial and final momenta of $\tilde{\nu}, T_{N}^{3}$ and $Q_{N}$ are the isospin and the electric charge of the nucleon, and $\theta_{W}$ is the Weinberg angle. For a slow-moving Dirac fermion of mass $M$ the leading terms in the $v / c$ expansion of the $u$ and $v$ spinors are (in Weyl representation)

$$
u(p)=\left(\begin{array}{c}
\left(1+\frac{\vec{p} \cdot \vec{\sigma}}{2 M_{p}}\right) \eta \\
\left(1-\frac{\vec{p} \cdot \vec{\sigma}}{2 M}\right) \eta
\end{array}\right), \quad v(p)=\left(\begin{array}{c}
\left(1+\frac{\vec{p} \cdot \vec{\sigma}}{2 M_{p}}\right) \eta \\
-\left(1-\frac{\vec{p} \cdot \vec{\sigma}}{2 M_{p}}\right) \eta
\end{array}\right)
$$

where $p$ is the fermion momentum and $\eta$ is a Weyl spinor. Using the above expressions one can explicitly verify that only the time component of the nucleon vector current survives in the limit $\vec{q} \rightarrow 0$. Therefore, one is left with a purely SI interaction, which is a special case of Eq. (15) with $f_{N}$ $=-\sqrt{2} G_{F}\left(T_{N}^{3}-2 Q_{N} \sin ^{2} \theta\right)$ and $a_{N}=0$.

\section{Spin 1/2}

In theories with extra dimensions Kaluza-Klein (KK) excitations of the SM particles can produce viable dark matter candidates. As an example, consider a Dirac KK neutrino, which interacts with nucleons via $Z^{0}$ exchange [13]:

$$
\mathcal{L}_{\nu}=-\sqrt{2} G_{F}\left(T_{N}^{3}-2 Q_{N} \sin ^{2} \theta_{W}\right) \bar{\nu}_{K K} \gamma_{\mu} \nu_{K K} \bar{N} \gamma^{\mu} N
$$

Just like in the previous example, only time components of currents remain in the nonrelativistic limit, and we again obtain [Eq. (15)] $f_{N}=-\sqrt{2} G_{F}\left(T_{N}^{3}-2 Q_{N} \sin ^{2} \theta_{W}\right)$ and $a_{N}$ $=0$.

\section{Spin-1}

Kaluza-Klein excitations of the SM gauge bosons could constitute cold dark matter [14]. If, for example, dark matter is composed of excitations of the hypercharge gauge boson $B_{1}$, the WIMP-quark interaction has the form

$$
\mathcal{L}_{B}=-\left(\beta_{q}+\gamma_{q}\right) B_{1}^{\dagger \mu} B_{1 \mu} \bar{q} q-i \alpha_{q} B_{1 \mu}^{\dagger} B_{1 \nu} \epsilon^{0 \mu \nu \rho} \bar{q} \gamma_{\rho} \gamma_{5} q,
$$

$$
\begin{aligned}
& \alpha_{q}=\frac{e^{2}}{2 \cos ^{2} \theta}\left[\frac{Y_{q L}^{2} m_{B_{1}}}{m_{q_{L}^{1}}^{2}-m_{B_{1}}^{2}}+(L \rightarrow R)\right], \\
& \beta_{q}=E_{q} \frac{e^{2}}{2 \cos ^{2} \theta}\left[Y_{q L}^{2} \frac{m_{B_{1}}^{2}+m_{q_{L}^{1}}^{2}}{\left(m_{q_{L}^{1}}^{2}-m_{B_{1}}^{2}\right)^{2}}+(L \rightarrow R)\right],
\end{aligned}
$$




$$
\gamma_{q}=m_{q} \frac{e^{2}}{4 \cos ^{2} \theta} \frac{1}{m_{h}^{2}},
$$

where $Y_{q}$ is the hypercharge of the quark with mass $m_{q}$ and energy $E_{q}, m_{q^{1}}$ is the mass of the quark's first KK excitation, and $m_{h}$ is the Higgs boson mass. The first term in Eq. (19) corresponds to the SI interaction in Eq. (15):

$$
\begin{aligned}
\langle N|\bar{q} q| N\rangle & =\frac{M_{N}}{m_{q}} f_{T_{q}}^{N} \bar{N} N=2 \frac{M_{N}}{m_{q}} f_{T_{q}}^{N} \eta_{N}^{\dagger} \eta_{N}, \\
f_{N} & =\frac{1}{2} \sum_{u, d, s, \ldots}\left(\beta_{q}+\gamma_{q}\right) \frac{M_{N}}{m_{q}} f_{T_{q}}^{N},
\end{aligned}
$$

where $f_{T_{q}}^{N}$ for various quark flavors can be found, e.g., in Refs. $[9,11]$ and $f_{N}$ is normalized to reproduce Eq. (15). The second term produces a SD WIMP-nucleon interaction:

$$
\begin{aligned}
i \epsilon^{0 i j k} B_{1 i}^{\dagger} B_{1 j}\left\langle N\left|\bar{q} \gamma_{k} \gamma_{5} q\right| N\right\rangle & =-4 \Lambda_{q}^{N}\left\langle B_{1}\left|\vec{S}_{B}\right| B_{1}\right\rangle \bar{N} \vec{S}_{N} N, \\
a_{N} & =\frac{1}{4 \sqrt{2} G_{F}} \sum_{u, d, s, \ldots} \alpha_{q} \Lambda_{q}^{N},
\end{aligned}
$$

where $\left(\hat{S}_{B}^{k}\right)_{i j}=i \epsilon^{0 k i j}$ is the matrix of the spin operator for $B_{1}, \Lambda_{q}^{N}$ is a dimensionless constant of order unity, and $a_{N}$ is normalized to reproduce Eq. (15). A recent analysis gives $\Delta_{u}^{p}=\Delta_{d}^{n}=0.78 \pm 0.02, \Delta_{d}^{p}=\Delta_{u}^{n}=-0.48 \pm 0.02$, and $\Delta_{s}^{p}=\Delta_{s}^{n}$ $=-0.15 \pm 0.02[15]$.

\section{WIMP-NUCLEUS CROSS SECTION}

The SI and SD WIMP-proton cross sections at low momentum transfers are easily calculated from Eq. $(15),{ }^{4}$

$$
\begin{aligned}
\sigma_{\chi p}^{S I} & =\frac{4 f_{p}^{2}}{\pi} M_{r e d}^{2}\left(M_{p}\right), \\
\sigma_{\chi p}^{S D} & =\frac{128 J_{\chi}\left(J_{\chi}+1\right) J_{p}\left(J_{p}+1\right) G_{F}^{2} a_{p}^{2}}{3 \pi} M_{r e d}^{2}\left(M_{p}\right), \\
M_{r e d}(M) & =\frac{M_{\chi} M}{M_{\chi}+M},
\end{aligned}
$$

where $M_{\chi}$ and $J_{\chi}$ are the WIMP mass and spin. In our convention, the nonrelativistic spinors $\psi$ for both the WIMP and nucleon are normalized as follows: $\psi_{\chi, p}^{\dagger} \psi_{\chi, p}=M_{\chi, p}$. The SI and SD WIMP-nucleus cross sections at asymptotically small energies, $\sigma_{\chi N}^{S I}$ and $\sigma_{\chi N}^{S D}$, can be expressed in terms of the corresponding WIMP-proton cross sections

${ }^{4}$ For a non-self-conjugate WIMP, such as the heavy Dirac neutrino, the WIMP-proton cross section must be multiplied by an extra factor of 1/4. This modification does not affect Eq. (24).

$$
\begin{aligned}
& \sigma_{\chi N}^{S I}=\frac{M_{r e d}^{2}\left(M_{n u c}\right)}{M_{r e d}^{2}\left(M_{p}\right)}\left[Z+(A-Z) \frac{f_{n}}{f_{p}}\right]^{2} \sigma_{\chi p}^{S I}, \\
& \sigma_{\chi N}^{S D}=\frac{M_{r e d}^{2}\left(M_{n u c}\right)}{M_{r e d}^{2}\left(M_{p}\right)} \frac{4(J+1)}{3 J}\left[\left\langle S_{p}\right\rangle+\left\langle S_{n}\right\rangle \frac{a_{n}}{a_{p}}\right]^{2} \sigma_{\chi p}^{S D} .
\end{aligned}
$$

Here $A$ and $Z$ are the mass number and the charge of the nucleus with spin $J$, and $f_{p}\left(f_{n}\right)$ and $a_{p}\left(a_{n}\right)$ are the SI and SD WIMP-proton (-neutron) couplings, respectively. The quantities $\left\langle S_{p}\right\rangle$ and $\left\langle S_{n}\right\rangle$ are the average spins of the proton and the neutron in the nucleus. At finite momentum transfer one must average the one-nucleon operators from Eq. (1) over the given nucleus using some nuclear structure model. Including the WIMP velocity distribution (see Ref. [9]), one then obtains, for the elastic scattering rate of WIMPs on the nucleus (per unit detector mass),

$$
\begin{aligned}
\frac{d R}{d E}= & \frac{\rho_{\chi}}{4 v_{E} M_{\chi} M_{r e d}^{2}\left(M_{n u c}\right)}\left[\operatorname{erf}\left(\frac{v_{\min }+v_{E}}{v_{0}}\right)\right. \\
& \left.-\operatorname{erf}\left(\frac{v_{\text {min }}+v_{E}}{v_{0}}\right)\right],\left[\sigma_{\chi N}^{S I} F_{S I}^{2}(E)+\sigma_{\chi N}^{S D} \frac{S_{A}(E)}{S_{A}(0)}\right], \\
v_{\text {min }}= & \sqrt{\frac{E M_{\text {nuc }}}{2 M_{r e d}^{2}}}
\end{aligned}
$$

where $\rho_{\chi}$ is the local Galactic halo density, ${ }^{5} E$ is the recoil energy of the nucleus with mass $M_{n u c}$, and $v_{E}$ and $v_{0}$ are, respectively, velocities of Earth and the Sun in the Galactic frame.

The form factors $F_{S I}(E)$ and $S_{A}(E)$ in Eq. (25) depend on the nuclear structure. The SI form factor can be well approximated by [11]

$$
\begin{aligned}
F_{S I}(E) & =\frac{3 j_{1}\left(q R_{1}\right)}{q R_{1}} e^{-(q s)^{2} / 2}, \\
q & =\sqrt{2 M_{n u c} E}, \\
R_{1} & =\sqrt{1.44 A^{2 / 3}-5 s^{2}} \mathrm{fm}, \\
s & \approx 1 \mathrm{fm},
\end{aligned}
$$

where $j_{1}$ is the spherical Bessel function. Unfortunately, there is no such universal expression for $S_{A}(E)$. It can be parametrized in terms of three nucleus-dependent functions:

$$
\begin{aligned}
S_{A}(E)= & \frac{1}{4}\left[\left(a_{p}+a_{n}\right)^{2} S_{00}(E)+\left(a_{p}-a_{n}\right)^{2} S_{11}(E)\right. \\
& \left.+\left(a_{p}^{2}-a_{n}^{2}\right) S_{01}(E)\right],
\end{aligned}
$$

where $S_{i j}(E)$ for most nuclei used in WIMP searches can be found in Ref. [16].

\footnotetext{
${ }^{5}$ We take $\rho_{\chi}=0.3 \mathrm{GeV} / \mathrm{cm}^{3}[9]$.
} 
It is apparent from Eqs. (24) and (25) that the detection rate for nonrelativistic WIMPs depends on only five parameters (in addition to nucleus-dependent constants). We choose these parameters to be $\sigma_{\chi p}^{S I}, \sigma_{\chi p}^{S D}, f_{n} / f_{p}, a_{n} / a_{p}$, and $M_{\chi}$. Various extensions of the SM generally reduce the number of parameters (e.g., in the MSSM one normally has $\left.f_{n} / f_{p} \approx 1[9]\right)$. In our analysis we do not impose such restrictions.

\section{EXPERIMENTAL DATA}

\section{A. DAMA NaI}

In utilizing the data published by the DAMA Collaboration in Ref. [2] we adopt the same approach as in Ref. [6]. In particular, we define the quantity

$$
\kappa=\sum_{i}\left[\frac{\left(S_{0, i}^{\text {th }}-S_{0, i}^{\exp }\right)^{2}}{\left(\Delta S_{0, i}^{\exp }\right)^{2}}+\frac{\left(S_{m, i}^{t h}-S_{m, i}^{\exp }\right)^{2}}{\left(\Delta S_{m, i}^{\exp }\right)^{2}}\right],
$$

where $S_{0, i}^{t h,(\exp )}$ and $S_{m, i}^{t h,(\exp )}$ are, respectively, the theoretical (experimental) average value and the annual-modulation amplitude of the detection rate in the $i$ th energy bin. Theoretical predictions for these quantities as functions of $M_{\chi}, \sigma_{\chi p}^{S I}$, $\sigma_{\chi p}^{S D}, f_{n} / f_{p}$, and $a_{n} / a_{p}$ can be obtained using Eq. (25). In this work, we used the experimental values for $S_{(0, m), i}^{\text {expt }}$ given in the first two columns of Table 1 in Ref. [2]. The DAMA preferred region, shown in Fig. 4(a) of Ref. [2], is well reproduced for $\kappa \approx 100$.

\section{B. DAMA Xe}

The latest results on WIMP searches with a liquid xenon target by the DAMA Collaboration have appeared in Ref. [7]. We take the limits on counts per detector per unit mass per day [detector rate unit (dru)] for various energy bins from Fig. 4 and Table I of this reference. In calculating the limits on the WIMP cross sections we follow the approach of Ref. [17]. We take the central values for the dru's in all energy bins to be zero and the $90 \%$ C.L. upper limits on the dru's to be equal to 1.3 times the total error bar on the dru's. We verified that the $90 \%$ C.L. upper limits appearing in the last column of Table I in Ref. [7] are reproduced in this way with the error bars from the second column of the same table.

In this approach, the 90\% C.L. upper limits on the dru in each energy bin result in an upper limit on the WIMPnulceon cross section $\sigma_{\chi N}^{\max }(k)$ with the help of Eq. (25). The combined upper limit from all energy bins is obtained using Eq. (15) of Ref. [17],

$$
\frac{1}{\left(\sigma_{\chi N}^{\max }\right)^{2}}=\sum_{k} \frac{1}{\left[\sigma_{\chi N}^{\max }(k)\right]^{2}} \text {. }
$$

We verified that our calculation reproduces the exclusion curves shown in Figs. 6(a) and 6(b) of Ref. [7] for both the SI and SD cases.

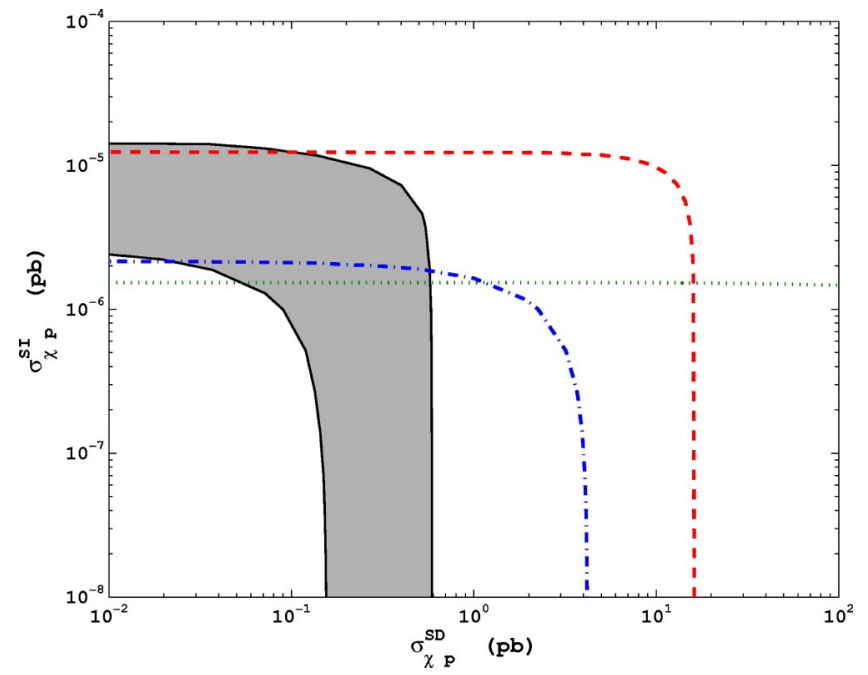

FIG. 1. Region in the $\sigma_{\chi p}^{S I}(\mathrm{pb})$ vs $\sigma_{\chi p}^{S D}(\mathrm{pb})$ plane allowed by various direct WIMP searches provided that the energetic neutrino constraint is not included. In this plot, $f_{n} / f_{p}=1, a_{n} / a_{p}=0$, and $M_{\chi}=50 \mathrm{GeV}$. Shaded region: DAMA/NaI. Dashed line: DAMA/ Xe129. Dotted line: EDELWEISS. Dash-dotted line: ZEPLIN-I. The region below the dotted line is allowed by all data.

\section{EDELWEISS}

We used Ref. [4] to incorporate the latest results from the EDELWEISS experiment that used a heat-and-ionization cryogenic Ge detector. In order to extract the $90 \%$ C.L. limit on the average expected number of events, $N$, from the null experimental result we used the Bayesian approach described in Ref. [1]. For our analysis, we adopt the following prior distribution for $N$ :

$$
\pi(N)= \begin{cases}0 & \text { for } N<0 \\ 1 & \text { for } N \geqslant 0\end{cases}
$$

which leads (after normalization) to a pair distribution function (PDF) for $N$ of the form $p_{0}(N)=e^{-N}$. Therefore, the probability for $N$ to be below some value $N_{0}$ is

$$
P_{0}\left(N<N_{0}\right) \equiv \int_{0}^{N_{0}} p_{0}(N) d N=1-e^{-N_{0}} .
$$

Equating the above probability to the confidence level of the EDELWEISS constraint $(90 \%)$, we obtain $N_{0}=\ln 10 \approx 2.3$.

The predicted number of events in the EDELWEISS detector for fixed values of WIMP parameters can be related to the detection rate by integrating Eq. (25) over the range of energies accepted by EDELWEISS (20-64 keV) and multiplying by the total effective exposure of $11.7 \mathrm{~kg} \cdot$ days [4]. In order to properly normalize the detection rate we multiply it by a factor $C_{E}$. This factor is adjusted such that the following predictions given in Ref. [4] for numbers of events are reproduced for the pure SI case: for $M_{\chi}=44 \mathrm{GeV}$ and $\sigma_{\chi p}^{S I}$ $=5.4 \times 10^{-6} \mathrm{pb}$ one has $N=6.2$, and for $M_{\chi}=52 \mathrm{GeV}$ and $\sigma_{\chi p}^{S I}=7.2 \times 10^{-6} \mathrm{pb}$ one has $N=9.8$. Note that since there is only one coefficient to determine, the second data point is redundant, and can thus be used for a consistency check. We find that for $C_{E} \approx 0.76$ both test points and the EDELWEISS 


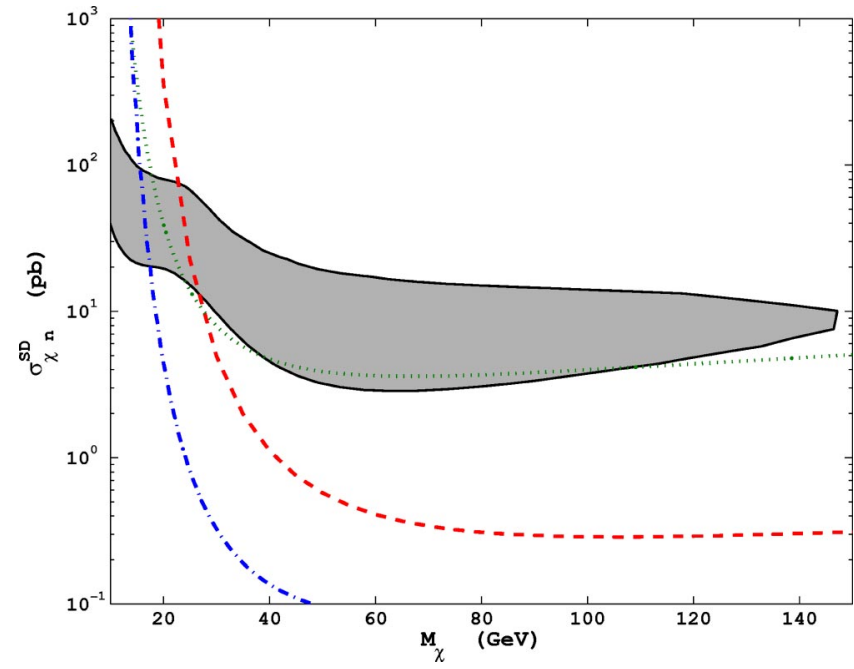

FIG. 2. Region in the $\sigma_{\chi n}^{S D}(\mathrm{pb})$ vs $M_{\chi}(\mathrm{GeV})$ plane allowed by various direct WIMP searches provided that the energetic neutrino constraint is included. We took $a_{p} / a_{n}=0$ (pure WIMP-neutron interaction). The meaning of various lines is as in Fig. 1.

exclusion curve given in Fig. 5 of Ref. [4] are well reproduced. In all the figures shown below, the constraints from EDELWEISS data are obtained by setting the predicted number of events in the detector equal to $N_{0}=\ln 10$.

\section{ZEPLIN-1}

The ZEPLIN-1 experiment (see Ref. [5]) used liquid xenon as a medium and relied on pulse-shape discrimination analysis. Unfortunately, at present there is no publication available containing detailed experimental results. The absence of such data prevented us from treating ZEPLIN-1 results in the same manner as those from the DAMA experiment with liquid Xe (see above). Instead, we chose an approach similar to the one we used for the EDELWEISS experiment. We deduced the parameters necessary for the calculation from Ref. [5]. In particular, we chose the visible energy to be between $4 \mathrm{keV}$ and $30 \mathrm{keV}$, with quenching factor $q_{X e}($ Zeplin $)=0.2$. These parameters correspond to recoil energies between $20 \mathrm{keV}$ and $150 \mathrm{keV}$. In order calibrate our calculation we introduced the effective statistics factor $C_{Z}$. We found that the SI 90\% CL ZEPLIN-1 limit shown in Ref. [5] is exceptionally well reproduced for $C_{Z}$ $\approx 8 \mathrm{~kg} \cdot$ days.

As a result of the absence of published results, the ZEPLIN-1 limit is the most uncertain input in our calculation. However, the results of our analysis are robust and are unlikely to change when the full data are properly included. Indeed, no change in the position of the ZEPLIN-1 curve in Fig. 2 will move the upper limit on the WIMP mass above about $25 \mathrm{GeV}$, which is still in conflict with the present lower limit on the MSSM lightest-neutralino mass of $37 \mathrm{GeV}$. Similarly, even if the ZEPLIN-1 constraint is entirely removed from the analysis, one would still require $f_{n} / f_{p}<0$ to achieve even marginal agreement among all data for the predominantly SI case (see caption to Fig. 3). As shown below $f_{n} / f_{p}<0$ would be very unusual in the MSSM.

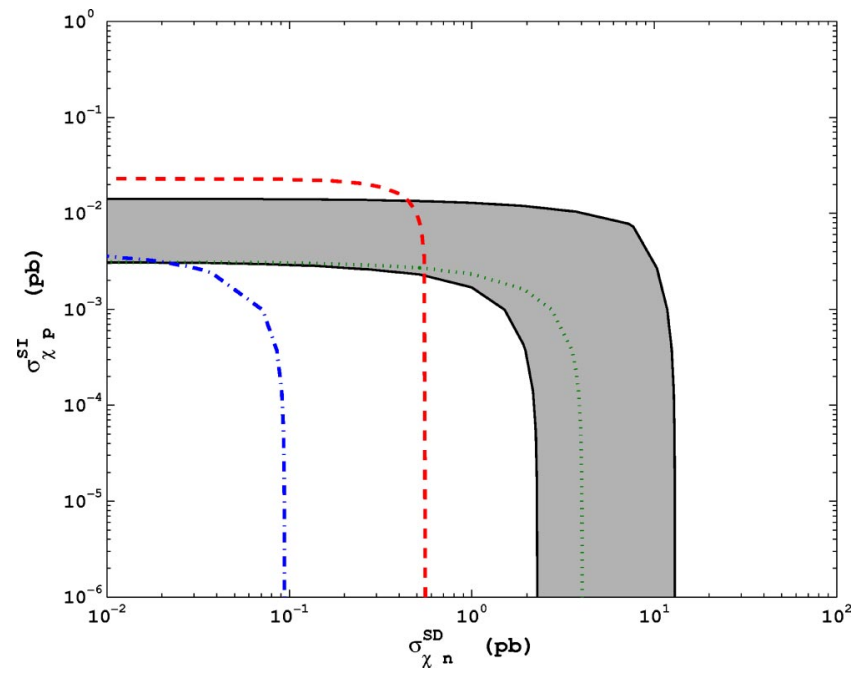

FIG. 3. Region in the $\sigma_{\chi p}^{S I}(\mathrm{pb})$ vs $\sigma_{\chi n}^{S D}(\mathrm{pb})$ plane allowed by various direct WIMP searches provided that the energetic neutrino constraint is included. We took $f_{n} / f_{p}=-0.76$. The meaning of various lines is as in Fig. 1.

\section{E. Energetic neutrino searches}

A promising method for indirect detection of WIMPs is the search for neutrinos from WIMP annihilation in the Sun and/or Earth. Such neutrinos produce upward muons in Earth via charged current interaction. Measurement of (or constraint on) the flux of such muons indirectly constrains the annihilation rate of WIMPs, which is related to the WIMP capture rate by the Sun and/or Earth. A detailed review of such indirect WIMP detection with an extensive list of references on the subject can be found in Ref. [9]. In this work, we use the limits on the fluxes of neutrino-induced upward muons near the surface of Earth obtained by SuperKamiokande [8]:

$$
\Gamma_{\mu}(\text { Sun }) \sim \Gamma_{\mu}(\text { Earth }) \lesssim 10^{-2} \mathrm{~m}^{-2} \cdot \mathrm{yr}^{-2} .
$$

Limits from other experiments, such as IMB, Baksan, MACRO, AMANDA, etc., are very similar (see Refs. [6,9] and references therein). The upward muon flux is related to the WIMP-proton cross section as

$$
\begin{aligned}
\Gamma_{\mu}^{S I}= & 1.96 \times 10^{-13} d \tanh ^{2}\left(\frac{t}{\tau}\right) \xi\left(M_{\chi}\right) f^{\prime}\left(M_{\chi}\right) \\
& \times\left(\frac{M_{\chi}}{\mathrm{GeV}}\right)^{2}\left(\frac{\sigma_{\chi p}^{S I}}{10^{-40} \mathrm{~cm}^{2}}\right), \\
\Gamma_{\mu}^{S D}(\operatorname{Sun})= & 1.6 \times 10^{-2} \tanh ^{2}\left(\frac{t}{\tau}\right) \xi\left(M_{\chi}\right) \\
& \times S\left(M_{\chi} / M_{p}\right)\left(\frac{M_{\chi}}{\mathrm{GeV}}\right)\left(\frac{\sigma_{\chi p}^{S D}}{10^{-40} \mathrm{~cm}^{2}}\right),
\end{aligned}
$$

where $d=3.3 \times 10^{8} \mathrm{~m}^{-2} \cdot \mathrm{yr}^{-2}$ for the Sun and $d=1.7$ $\times 10^{8} \mathrm{~m}^{-2} \cdot \mathrm{yr}^{-2}$ for Earth, and $\tau$ is the time scale for equilibration between WIMP capture and WIMP annihilation. For 
the Sun, we can take $\tanh (t / \tau) \approx 1$, whereas for Earth it is likely substantially smaller than unity [9]. The function $\xi(M)$ is given by Eq. (9.54) and $S(x)$ by Eq. (9.21) in Ref. [9]. The function $f^{\prime}(M)$ is the generalization of the function $f(M)$ given by Eq. (9.28) of the same reference:

$$
\begin{aligned}
f^{\prime}(M)= & \sum_{i} f_{i} \phi_{i} S\left(M_{\chi} / M_{n u c}^{i}\right) F_{i}\left(M_{\chi}\right) \frac{M_{n u c}^{i 3} M_{\chi}}{M_{p}^{2}\left(M_{\chi}+M_{n u c^{i}}\right)^{2}} \\
& \times\left[\frac{Z_{i}}{A_{i}}+\left(1-\frac{Z_{i}}{A_{i}}\right) \frac{f_{n}}{f_{p}}\right]^{2}
\end{aligned}
$$

where the sum runs over the nuclei with mass $M_{n u c}^{i}$, charge $Z_{i}$, and atomic number $A_{i}$. The quantities $f_{i}$ and $\phi_{i}$ are given in Tables 8 and 9 of Ref. [9]. The form-factor suppression $F_{i}\left(M_{\chi}\right)$ is only important for iron in the Earth, where it is given by Eq. (9.23) of Ref. [9].

One can check that for $M_{\chi} \gg M_{p}$ the muon flux $\Gamma_{\mu}^{S D}$ is independent of $M_{\chi}$, which leads to the constraint $\sigma_{\chi p}^{S D}<1.2$ $\times 10^{-4} / \xi \mathrm{pb}$. In the MSSM one generally has $\xi \geqslant 0.03$ [9], and we obtain $\sigma_{\chi p}^{S D}<4 \times 10^{-3} \mathrm{pb}$. The constraints on $\sigma_{\chi p}^{S I}$ from energetic solar neutrinos and on $\sigma_{\chi(p, n)}^{S D}$ from terrestrial neutrinos are too weak to be interesting. On the other hand, the constraint on $\sigma_{\chi p}^{S I}$ from energetic neutrinos originating in the Earth is potentially nontrivial. For the case $f_{n} / f_{p}$ $\approx-0.75$ and $M_{\chi} \approx 50 \mathrm{GeV}$ (see Sec. V B) one obtains

$$
\sigma_{\chi p}^{S I}<\frac{3.6 \times 10^{-6}}{\xi \tanh ^{2}\left[t / \tau_{\oplus}\right]} \mathrm{pb}
$$

As shown below, one needs $\sigma_{\chi p}^{S I} \approx 0.0035 \mathrm{pb}$ for this case. In addition, one can deduce, from Ref. [18],

$$
\left(\frac{t}{\tau_{\oplus}}\right)^{2}=2.4 \times 10^{3} f^{\prime}(50 \mathrm{GeV}) \frac{\sigma_{A} v}{10^{-26} \mathrm{~cm}^{3} \cdot \mathrm{s}^{-1}} \frac{\sigma_{\chi p}^{S I}}{1 \mathrm{pb}}
$$

where $\sigma_{A}$ is the WIMP annihilation cross section in the limit of zero relative WIMP velocity $v$. In order to have WIMP relic density of order 1 we must have $\sigma_{A} v \approx 10^{-26}$ $\mathrm{cm}^{3} \cdot \mathrm{s}^{-1}$ [18], and for $f_{n} / f_{p}=-0.76$ one has $f^{\prime}(50 \mathrm{GeV})$ $=0.85$. Therefore, $\left(t / \tau_{\oplus}\right)^{2} \approx 2 \times 10^{3}\left(\sigma_{\chi p}^{S I} / 1 \mathrm{pb}\right) \approx 8$ or $\tanh ^{2}\left(t / \tau_{\oplus}\right) \approx 1$. Now, Eq. (35) can be simply rewritten as a constraint on $\xi$ : $\xi<0.001$ for $f_{n} / f_{p}=-0.76$.

Can the constraints coming from the nonobservation of upward muons from energetic solar neutrinos be evaded? Below we consider two possibilities. In the first case, the MSSM WIMPs predominantly decay into light fermions. Because the annihilation rate $\Gamma\left(\chi \chi \rightarrow f^{+} f^{-}\right)$is proportional to the fermion mass squared, $m_{f}^{2}$, direct annihilation into neutrinos is virtually impossible, and energetic neutrinos appear in the decay chain of the initial annihilation products. The total flux of the neutrinos originating from the branch $\Gamma\left(\chi \chi \rightarrow f^{+} f^{-}\right)$inherits the suppression by $m_{f}^{2}$, and in the case where only light fermions appear during annihilation the flux may be orders of magnitude below the conventional estimates [6]. Effectively, in this case $\xi\left(M_{\chi}\right)$ is substantially smaller than estimated in Ref. [9], which weakens the constraints on $\sigma_{\chi p}^{S I, S D}$. In the second case, the WIMP is not identical to the anti-WIMP, and only WIMP-anti-WIMP annihilation is allowed. Although direct annihilation into neutrinos may be possible (leading to stronger energetic neutrino signals), the annihilation rate may still be significantly suppressed in the presence of significant WIMP-anti-WIMP asymmetry.

\section{WIMPs only decay into light fermions}

As pointed out in Ref. [6] the energetic neutrino constraint could be evaded if the WIMPs annihilated to $u \bar{u}, d \bar{d}$, $s \bar{s}, e^{+} e^{-}$, and/or $\mu^{+} \mu^{-}$pairs but not $c \bar{c}, b \bar{b}$, or $\tau \bar{\tau}$ pairs. Such a situation can in principle be achieved in the MSSM by fine-tuning the sfermion masses and WIMP composition. Let us write the lightest-neutralino field as

$$
\chi=Z_{1} \widetilde{B}+Z_{2} \widetilde{W}_{3}+Z_{3} \widetilde{H}_{1}+Z_{4} \widetilde{H}_{2}
$$

where $Z_{i}$ are constants subject to $\Sigma\left|Z_{i}\right|^{2}=1, \widetilde{B}$ and $\widetilde{W}_{3}$ are superpartners of the $B$ and $W_{3}$ gauge bosons, and $\widetilde{H}_{1,2}$ are superpartners of the neutral Higgs bosons. Examination of the general expressions for the axial-vector and scalar neutralino-fermion couplings [Eq. (3.6) in Ref. [11]] shows that if $\left|Z_{3}\right|=\left|Z_{4}\right|$ and $Z_{2}=\tan \theta_{W} Z_{1}$, the scalar coupling vanishes and the axial coupling $a_{f}$ is inversly proportional to the fermion superpartner mass squared:

$$
a_{f} \sim \frac{1}{M_{\tilde{f}}^{2}}
$$

where $M_{\tilde{f}}$ is the mass of the superpartner of the fermion $f$. Choosing the superpartner mass to be very large for the charm and bottom quarks, as well as for the $\tau$ lepton, one can force the neutralinos to annihilate into light quarks and leptons only. However, choosing such flavor nonuniversal masses for the scalars may be problematic in the MSSM. Indeed, one must obey the existing stringent constraints on the size of the flavor-changing neutral current (FCNC) (see, e.g., Ref. [19] for a review of FCNC constraints on the MSSM spectrum). Since evading energetic neutrino constraints in a way we just described requires significant flavor nonuniversality among certain entries of the squark mass matrices, whereas the smallness of the FCNC prefers the opposite, one may expect that fine-tuning would be required to make any such scenario phenomenologically viable. We note that even for non-MSSM WIMPs flavor nonuniversality of the WIMP couplings is needed to evade the energetic neutrino constraint in the described manner, and experimental limits on FCNC are likely to present complications in for any WIMP candidate.

\section{WIMPs are not identical to anti-WIMPs}

Another way to evade the energetic neutrino constraint is to consider WIMPs that are not identical to their antiparticles. Indeed, for the total number density of dark matter particles, $N_{0}$, one has 


$$
\begin{aligned}
& n_{0}+\bar{n}_{0}=N_{0}, \\
& n_{0}-\bar{n}_{0}=W,
\end{aligned}
$$

where $n_{0}$ and $\bar{n}_{0}$ are the WIMP and anti-WIMP densities in the vicinity of the Solar System, and $W$ is the "WIMP number" density, which may be generated in presence of some $T$-, $C$-, and $C P$-violating physics. The (anti-)WIMP densities $n$ and $\bar{n}$ inside the solar core obey the equations [20]

$$
\begin{aligned}
& \dot{n}=A n_{0}-B n \bar{n}-\Gamma_{e s c}, \\
& \dot{\bar{n}}=\bar{A} \bar{n}_{0}-B n \bar{n}-\bar{\Gamma}_{e s c},
\end{aligned}
$$

where the first and second terms on the right-hand side (RHS) of both equations represent, respectively, the capture rate of WIMPs incident on the Sun and the annihilation of WIMPs and anti-WIMPs in the solar core. The constants $A$ and $\bar{A}$ are proportional, respectively, to the WIMP-proton and anti-WIMP-proton scattering cross sections. The last terms represent the losses of WIMPs due to evaporation (see, e.g., Ref. [21]).

Consider the case where $n_{0} \gg \bar{n}_{0}$. In the stationary state $\dot{\bar{n}}=0$, and we have for the annihilation rate $\Gamma_{a n n}=\bar{A} \bar{n}_{0}$. We make a reasonable assumption that annihilation is the dominant mechanism for anti-WIMP loss if $\bar{n} \ll n$ because the evaporation rate is suppressed by a small Boltzman-like factor $e^{-E_{e} / k T_{W}}$. Here, $E_{e}$ is the escape energy and $T_{W}$ is the effective WIMP temperature. On the other hand, for neutralinos in the MSSM we have $\left(\Gamma_{\text {esc }}\right.$ can be neglected here as well)

$$
\dot{N}=A^{M S S M} N_{0}-B^{M S S M} N^{2} .
$$

In the stationary state this would give for the annihilation rate $\Gamma_{a n n}^{M S S M}=A^{M S S M} N_{0}$. We find that

$$
\Gamma_{a n n}=\frac{\bar{A}}{A^{M S S M}} \frac{\bar{n}_{0}}{N_{0}} \Gamma_{a n n}^{M S S M}=\frac{\bar{A}}{2 A^{M S S M}} \Gamma_{a n n}^{M S S M}\left(1-\frac{W}{N_{0}}\right) .
$$

We generally expect $A \sim \bar{A}$, and since the WIMP-proton cross section is constrained by DAMA, we expect $A$ $\sim A^{M S S M}$. Therefore, we conclude that $\Gamma_{\text {ann }} \sim \Gamma_{\text {ann }}^{M S S M}(1$ $\left.-W / N_{0}\right)$. The flux of upward muons in the Super Kamiokande detector is proportional to the WIMP annihilation rate: $\Gamma_{\mu} \sim \xi\left(M_{\chi}\right) \Gamma_{a n n}$. Therefore, we obtain

$$
\Gamma_{\mu} \sim \frac{\xi\left(M_{\chi}\right)}{\xi^{M S S M}\left(M_{\chi}\right)}\left(1-\frac{W}{N_{0}}\right) \Gamma_{\mu}^{M S S M}
$$

Since the WIMP is not identical to its anti-WIMP, direct WIMP annihilation into neutrinos may be possible, which may yield $\xi\left(M_{\chi}\right) / \xi^{M S S M}\left(M_{\chi}\right)>1$. On the other hand, for
$1-W / N_{0} \ll 1$, the muon detection rate in the Sun can still be significantly below the one predicted by Eq. (5) in Ref. [6], and the energetic neutrino constraint may not apply. In this work, we first perform the analysis without this constraint and then add it later on.

\section{ANALYSIS}

\section{A. Energetic neutrino constraints are not applicable}

Let us first consider the constraints on the WIMP parameter space without the energetic neutrino bounds. In this case an agreement between all direct search experiments can be achieved for the predominantly SD WIMP-nucleon interaction for a wide range of WIMP masses and couplings. A similar conclusion was originally obtained in Ref. [6]. In Fig. 1 we plot the allowed region in the $\sigma_{\chi p}^{S I}$ vs $\sigma_{\chi p}^{S D}$ plane for $f_{p} / f_{n}=1, a_{n} / a_{p}=0$, and $M_{\chi}=50 \mathrm{GeV}$. The shaded region is allowed by all direct search experiments described in Sec. IV. Since a substantial SD interaction is required, the WIMP cannot be a scalar particle in this case.

\section{B. Energetic neutrino constraints are applicable}

We now include the energetic neutrino constraint. As is well known, for the usual case $f_{n} / f_{p} \approx 1$ the results from all direct search experiments cannot be reconciled for the pure SI case at $3 \sigma$ level. With the WIMP-proton SD cross section limited from above by the energetic neutrino searches, a relatively large WIMP-neutron SD cross section is needed to accommodate the DAMA/NaI result. The situation is similar to the one described in Ref. [6] for the pure WIMP-neutron interaction, and the allowed region for this case is shown in Fig. 2. For this figure, we kept the WIMP-proton SD cross section close to its upper limit set by the Super-Kamiokande search; reducing this cross section increases the required SD WIMP-neutron cross section and, therefore, strengthens the upper limit on the WIMP mass.

The strongest upper limit, about $18 \mathrm{GeV}$, will likely be provided by the ZEPLIN-1 experiment. We found that this limit does not significantly change even if some SI interaction is allowed. If the ZEPLIN-1 result is not included, the upper limit on the WIMP mass increases to about $25 \mathrm{GeV}$.

It is interesting to note that MSSM neutralinos with mass below $37 \mathrm{GeV}$ are excluded by direct collider searches [1]. Although the analysis of such searches is not completely general, since it assumes gaugino and sfermion mass unification at the grand unified theory (GUT) scale [1], evading this limit, if at all possible, would require fine-tuning of the MSSM parameters.

$\mathrm{Up}$ to this point, we always maintained the condition $f_{n} / f_{p}=1$. It turns out that relaxing this constraint allows one to achieve marginal agreement among all data for $M_{\chi}$ in the vicinity of $50 \mathrm{GeV}$. However, with the ZEPLIN-1 result included this occurs for only a very narrow range of $f_{n} / f_{p}$. Specifically, we need $-0.77 \lesssim f_{n} / f_{p} \lesssim-0.75$. If ZEPLIN-1 is not considered, the allowed range is increased but the agreement is still marginal. The situation for $f_{n} / f_{p}=-0.76$ is shown in Fig. 3. The small region allowed by all data is centered around $\sigma_{\chi p}^{S I} \approx 0.0035 \mathrm{pb}$. Note that, as a result of a 
significant cancellation between the WIMP-neutron and WIMP-proton scattering amplitudes, the individual WIMPnucleon cross sections are about $10^{2}$ times larger than in the case where $f_{n} / f_{p}=1$.

The inverse of this number, 0.01 , reflects the amount of fine-tuning required for this solution to work. Marginal agreement is possible to achieve because the proton to neutron ratio varies only slightly from one nucleus to another, and it is possible to choose $f_{n} / f_{p}$ such that all constraints are satisfied.

From standpoint of the MSSM, this solution is in fact "doubly fine-tuned." In addition to making sure that $f_{n} / f_{p}$ is carefully selected to fit all data one has to tune the model of SUSY breaking to even obtain $f_{n} / f_{p}<0$ (normally, one has $f_{n} \approx f_{p}$ in the MSSM [9]). It is interesting to ask, therefore, whether a significant deviation from the approximate equality $f_{n} / f_{p} \approx 1$ is at all possible within the MSSM. In general, we have

$$
f_{N}=f_{0}+f_{1} \hat{\tau}_{3}
$$

where $N=p, n$ and $\hat{\tau}_{3}$ is the usual Pauli matrix in the strong isospin space. The Feynman diagrams and detailed expressions for $f_{p, n}$ can be found in Ref. [9]. Since only the up and down quarks have nonzero strong isospin, the isovector part of $f_{N}$ is entirely due to coupling of the neutralino bilinear to isovector operators built from the up and down quarks. The isoscalar part arises due to coupling to various isoscalar operators built from the up and down quarks, the strange and all heavy quarks, and the gluons. The scalar coupling of the neutralino to a quark has the form ${ }^{6}[9]$

$$
\begin{aligned}
\mathcal{L}_{\chi, q}= & G_{F} \frac{m_{q}}{M_{W}} \bar{\chi} \chi \bar{q} q\left(A_{q} \frac{M_{W}^{2}}{M_{h}^{2}}+B_{q} \frac{M_{W}^{2}}{M_{\tilde{q}}^{2}-\left(M_{\chi}+m_{q}\right)^{2}}\right) \\
& +\mathcal{O}\left(\frac{1}{M_{\tilde{q}}^{4}}\right) \approx m_{q} f_{q} \bar{\chi} \chi \bar{q} q,
\end{aligned}
$$

where $G_{F}$ is the Fermi constant, $A_{q}$ and $B_{q}$ are dimensionless constants, $m_{q}$ is the quark mass, $M_{\tilde{q}}$ is the mass of the superpartner of $q, M_{h}$ is the lightest Higgs-boson mass, and $M_{\chi}$ is the neutralino mass. We can now write the couplings $f_{p, n}$ in the form [9]

$$
f_{p, n}=m_{p, n}\left[f_{T u}^{p, n} f_{u}+f_{T d}^{p, n} f_{d}+f_{T s}^{p, n} f_{s}+\cdots\right],
$$

where "..." stands for both the isovector terms of order $1 / M_{\tilde{q}}^{4}$ and all remaining isoscalar contributions. Here, $m_{N} f_{T q}^{N}=\left\langle N\left|m_{q} \bar{q} q\right| N\right\rangle$. Ignoring the remaining terms we obtain (see Table 6 in Ref. [9])

\footnotetext{
${ }^{6} \mathrm{We}$ assume that the squark mass matrices are diagonal in the flavor space.
}

$$
\begin{aligned}
\left|\frac{f_{1}}{f_{0}}\right| & \lessgtr\left|\frac{f_{u}\left(f_{T u}^{p}-f_{T u}^{n}\right)+f_{d}\left(f_{T d}^{p}-f_{T d}^{n}\right)}{2 f_{T s} f_{s}+f_{u}\left(f_{T u}^{p}+f_{T u}^{n}\right)+f_{d}\left(f_{T d}^{p}+f_{T d}^{n}\right)}\right| \\
& \approx\left|\frac{-0.014 f_{u}+0.025 f_{d}}{f_{s}+0.15 f_{u}+0.27 f_{d}}\right| .
\end{aligned}
$$

In most models of SUSY breaking $f_{u, d} \sim f_{s}$, and although the above range is nothing more than an estimate, it at least shows that $\left|f_{1} / f_{0}\right| \geqslant 1$ (which would lead to a negative ratio $\left.f_{n} / f_{p}\right)$ is generically disfavored in the MSSM. However, it is possible in principle to fine-tune the parameters to obtain $\left|f_{1} / f_{0}\right| \geqslant 1$. One scenario arises when $\left|f_{s}\right| \ll\left|f_{u, d}\right|$. This can be achieved if $M_{\tilde{q}}$ is very large for all squarks except for $\tilde{u}$ and $\widetilde{d}$, all physical Higgs bosons, except the lightest one, are very heavy, and the coupling between the WIMP and lightest Higgs boson is tuned to zero. In this case, one finds (we use the MSSM Feynman rules given in Ref. [22] and bring the notation in correspondence with Ref. [11])

$$
\begin{aligned}
& f_{u}=-\frac{g^{2}}{4 M_{W} M_{\tilde{u}}^{2} \sin \beta} Z_{4}\left(Z_{2}-\tan \theta_{W} Z_{1}\right), \\
& f_{d}=\frac{g^{2}}{4 M_{W} M_{\tilde{d}}^{2} \cos \beta} Z_{3}\left(Z_{2}-\tan \theta_{W} Z_{1}\right), \\
& f_{s} \approx 0 .
\end{aligned}
$$

Here $g$ is the $\mathrm{SU}(2)_{L}$ gauge coupling and $\tan \beta$ is the ratio of the expectation values for the two Higgs boson doublets in the MSSM. We find

$$
\left|\frac{f_{1}}{f_{0}}\right| \sim\left|\frac{0.014+0.25 \tan ^{2} \beta \frac{M_{\tilde{u}}^{2}}{M_{\tilde{d}}^{2}}}{-0.15+0.27 \tan ^{2} \beta \frac{M_{\tilde{u}}^{2}}{M_{\tilde{d}}^{2}}}\right| .
$$

If $M_{\tilde{d}} / M_{\tilde{u}} \approx 1.3 \tan \beta$, then one can have $\left|f_{1} / f_{0}\right|>1$, which may lead to $f_{n} / f_{p}<0$. At present, $\tan \beta \gtrsim 3$ is favored by precision data [1]. Therefore, one needs a mild hierarchy between the up- and down-squark masses to obtain $f_{n} / f_{p}$ $<0: M_{\tilde{d}} \geqslant 3.9 M_{\tilde{u}}$.

There might be other scenarios yielding $f_{n}$ substantially different from $f_{p}$. However, they will all have to share the same property: the first generation of squarks must be singled out from the rest to enhance the up- and down-quark contributions to $f_{0,1}$. Reconciling such significant flavor nonuniversality of the squark flavors with stringent constraints from FCNC will generally require fine-tuning. In this sense, having significantly different values for $f_{n}$ and $f_{p}$ is unnatural, although potentially possible, in the MSSM. There is an additional complication, however. For this scenario to work, the WIMP-proton scattering cross section must be roughly 100 times larger than for the case $f_{n} \approx f_{p}$. On the other hand, one must have $\xi<0.001$ in this case (requiring $Z_{2}-\tan \theta_{W} Z_{1} \ll 1$; see Sec. IV E 1 ), which suppresses $f_{u, d}$ 
according to Eq. (48). Partly as a result of this suppression, we have found no scenario where sufficient enhancement of $f_{p, n}$ would occur in the MSSM for $M_{\chi} \approx 50 \mathrm{GeV}$ together with $f_{n} / f_{p} \approx-0.75$.

We point out that $f_{n} / f_{p} \approx-0.75$ does not hold for any of the dark-matter candidates we considered. For scalar neutrinos and KK neutrinos one finds (see Sec. II ) $f_{n} / f_{p}$ $=T_{n}^{3} /\left(T_{p}^{3}-2 Q_{p} \sin ^{2} \theta_{W}\right) \approx-10$. A detailed study of this ratio for KK excitation of the hypercharge gauge boson shows that it is at most few percent away from unity. In summary, although there exists a small phenomenologically allowed region of parameter space where predominantly SI WIMPnucleon interactions can marginally account for all data available on WIMP searches, this region appears to be out of reach for all of the WIMP candidates we considered.

\section{CONCLUSIONS}

In this work we performed a generalized analysis of the dark-matter detection experiments listed in Sec. IV. Our analysis is formulated in terms of the WIMP mass $M_{\chi}$, the SI and SD WIMP-proton cross sections $\sigma_{\chi p}^{S I, S D}$, and coupling ratios $f_{n} / f_{p}$ and $a_{n} / a_{p}$. We found several regions in this parameter space that allow for agreement among all data.

(i) If the energetic neutrino constraint does not apply, it is possible to reconcile all data with a predominantly SD WIMP-proton interaction. Evading the constraint is problematic in the MSSM because it requires highly flavor nonuniversal squark masses. Another possibility is a non-selfconjugate WIMP, which would indicate physics other than the MSSM. In order for this possibility to be realistic, such physics must accommodate WIMP-number-violating operators and possess a sufficient amount of $C$ and $C P$ violation to generate a fractional WIMP asymmetry near unity.

(ii) If the energetic neutrino constraint applies, then the SD WIMP-proton cross section is constrained to be $\sigma_{\chi p}^{S D}$ $\leqslant 4 \times 10^{-3} \mathrm{pb}$ in the MSSM, and for a wide range of $f_{n} / f_{p}$, a SD WIMP-neutron cross section $\sigma_{\chi n}^{S D} \geqslant 30 \mathrm{pb}$ is required. In addition, $M_{\chi}$ is constrained to be below $18 \mathrm{GeV}$, which is inconsistent with present lower limit on the lightestneutralino mass within most models of SUSY breaking [1]

(iii) A heavier WIMP (about $50 \mathrm{GeV}$ ) is allowed for a narrow range $-0.77 \leq f_{n} / f_{p} \lesssim-0.75$ with essentially no SD interaction present. In addition, one must have $\sigma_{\chi p}^{S I}$ $\approx 0.0035 \mathrm{pb}$. None of the WIMP candidates we considered could satisfy both requirements at the same time.

We see that although it is possible to reconcile the direct and indirect dark-matter search experiments listed in Sec. IV, the resulting parameters are in general unnatural for the MSSM with most used models of SUSY breaking. Either significant flavor nonuniversality among the SUSY breaking parameters (e.g., to evade the energetic neutrino constraint) or some delicate relationships among them (to avoid the lower limit on the lightest neutralino mass) appear necessary. Generating the WIMP parameters in any of the allowed regions may be problematic in a theory with non-MSSM WIMPs as well. For instance, evading the energetic neutrino constraint in a theory with non-self-charge-conjugate WIMPs may require a significant amount of $C P$ violation to generate a fractional WIMP number close to 1 . Such $C P$-violating interactions may be strongly constrained by existing limits on, e.g., neutron and atomic electric dipole moments [1]. If the energetic neutrino constraint is adopted, one would have to explain how very light WIMPs $\left(M_{\chi} \lesssim 18 \mathrm{GeV}\right)$ with significant WIMP-quark couplings have so far escaped detection.

On the other hand, if the DAMA result is removed from the analysis, the MSSM neutralino (as well as other WIMP candidates) will be compatible with the remaining experiments and still be capable of being produced with sufficient abundance to account for all dark matter [1]. In view of this situation, one can hope that forthcoming direct dark-matter search experiments, potentially capable of improving the present sensitivity by several orders of magnitude, will be able to conclusively confirm or exclude the results published by DAMA based on their observation of annual modulation of the event detection rate.
[1] Particle Data Group, K. Hagiwara et al., Phys. Rev. D 66, 010001 (2002).

[2] DAMA Collaboration, R. Bernabei et al., Phys. Lett. B 480, 23 (2000); R. Bernabei et al., Riv. Nuovo Cimento 26, 1 (2003).

[3] CDMS Collaboration, R. Abusaidi et al., Phys. Rev. Lett. 84, 5699 (2000).

[4] EDELWEISS Collaboration, A. Benoit et al., Phys. Lett. B 545, 43 (2002).

[5] N.J.T. Smith, talk at idm2002, 4th International Workshop on Identification of Dark Matter, http://www.shef.ac.uk/ phys/ idm2002/talks/pdfs/smith_n.pdf; N.J.C. Spooner, talk at The 3rd Workshop on "Neutrino Oscillations and their Origin" (NOON2001), http://www-sk.icrr.u-tokyo.ac.jp/noon2001/ transparency/dec03/spooner/spooner.pdf

[6] P. Ullio, M. Kamionkowski, and P. Vogel, J. High Energy Phys. 07, 044 (2001).
[7] DAMA Collaboration, R. Bernabei et al., Phys. Lett. B 436, 379 (1998).

[8] Super-Kamiokande Collaboration, A. Okada et al., astro-ph/0007003.

[9] G. Jungman, M. Kamionkowski, and K. Griest, Phys. Rep. 267, 195 (1996).

[10] A.M. Green, Phys. Rev. D 68, 023004 (2003); P. Belli, R. Cerulli, N. Fornengo, and S. Scopel, ibid. 66, 043503 (2002); C.J. Copi and L.M. Krauss, ibid. 67, 103507 (2003); N. Fornengo and S. Scopel, hep-ph/0301132.

[11] J. Engel, S. Pitte, and P. Vogel, Int. J. Mod. Phys. A 1, 1 (1992).

[12] Mark W. Goodman and Edward Witten, Phys. Rev. D 31, 3059 (1985).

[13] T. Appelquist, H.-C. Cheng, and B.A. Dobrescu, Phys. Rev. D 64, 035002 (2001). 
[14] H.-C. Cheng, J.L. Feng, and K.T. Matchev, Phys. Rev. Lett. 89, 211301 (2002).

[15] G.K. Mallot, Int. J. Mod. Phys. A 15, S1 521 (2000).

[16] M.T. Ressell and D.J. Dean, Phys. Rev. C 56, 535 (1997).

[17] P.F. Smith et al., Phys. Lett. B 379, 299 (1996).

[18] M. Kamionkowski, K. Griest, G. Jungman, and B. Sadoulet,
Phys. Rev. Lett. 74, 5174 (1994).

[19] F. Gabbiani, E. Gabrielli, A. Masiero, and L. Silvestrini, Nucl. Phys. B477, 321 (1996).

[20] K. Griest and D. Seckel, Nucl. Phys. B283, 681 (1986).

[21] A. Gould, Astrophys. J. 321, 560 (1987).

[22] J. Rosiek, hep-ph/9511250. 